\title{
Electroencephalography-guided Upper-Limb Hybrid Robotic Platform to Modulate Cortical Excitability
}

\author{
F. Resquín ${ }^{1}$, O. Herrero ${ }^{1}$, J. Ibañez ${ }^{2}$, J. Gonzalez-Vargas ${ }^{1}$, F. Brunetti ${ }^{3}$, J. L. Pons ${ }^{1}$ \\ ${ }^{1}$ Neural Rehabilitation Group, Cajal Institute. Madrid, Spain. \\ ${ }^{2}$ Sobell Deparment, Institue of Neurology, University College London. London, UK \\ ${ }^{3}$ Catholic University of Asunción, Paraguay.
}

\begin{abstract}
This study present an intervention combining an electroencephalography-based brain computer interface with a hybrid robotic system for the modulation of the cortical excitability (plasticity). Plasticity is intended to be elicited through the association of the voluntary motor-related cortical processes with the hybrid assistance during the execution of reaching movement. The cortical excitability was assessed before and after the intervention measuring the peak-to-peak amplitude of the Motor Evoked Potentials (MEPs) induced through transcranial magnetic stimulation pulses. Five healthy subjects participated in the experiments. Results showed an overall and distributed increase in the cortical excitability as a result of the proposed intervention.
\end{abstract}

Keywords-BCI; FES; Reaching movement; Plasticity; Rehabilitation;

\section{INTRODUCTION}

Different novel rehabilitation therapies have been proposed over the past years to improve the current rehabilitation outcomes in subjects with a lesion on the central nervous system, like stroke or spinal cord injury. Robotic devices [1], functional electrical stimulation (FES) technologies [2], and the combined use of these solutions in so-called hybrid robotic systems [3] are of particular relevance due to their expected impact on the future of neurorehabilitation [4]. Although most studies presented in the literature regarding these technologies focused on evaluating the improvement of motor function in patients, little evidences have been provided regarding the potential impact of rehabilitation technologies for the improvements of neural connections (plasticity) between the brain and the peripheral muscles in the body. In this regard it is worth noting that after a neurological lesion, like stroke, facilitating neural plasticity plays an important role in the recovery of motor function [5].

Under this scope, in a series of studies, the use of BrainComputer Interfaces (BCI) as neuromodulation system has been proposed. In the rehabilitation field, a neuromodulation BCI can be defined as a system conceived for inducing neuroplasticity [6]. In this regard, there are several pieces of evidence showing that timed-association between voluntary motor-related cortical patterns and peripheral afferent feedback yields a well-adaptive plasticity [7].

Between these studies, Mrachacz-Kersting et al. have demonstrated that applying a peripheral electrical stimulus over the peroneal nerve timed with the negative peak of the contingent negative variation results in an increased excitability of the cortical projections to the tibialis anterior muscle when assessed with Transcranial Magnetic Stimulation (TMS) in healthy subjects [8]. Remarkably, they have also demonstrated that this plasticity is correlated with motor functional improvements of the lower limb in stroke patients [9]. In this line, $\mathrm{Xu}$ et al. replaced the electrical stimulus with a robotic device providing mechanical support to execute the same ankle dorsi-flexion task in heathy subjects, and obtained similar results [10]. Despite these promising results, in these studies the number of muscles involved in the tasks was reduced (single muscle for one degree of freedom) and involved analytical tasks instead of functional ones.

Likewise, some studies addressed the same approach (BCI + peripheral afferent feedback) to corroborate cortical plastic changes when executing grasping movement assisted by FES [11] and a robotic device [12]. Although the functional connotation of this task (grasping) is high (from the rehabilitation perspective), both studies failed to demonstrate an increased excitability of the cortical projections to the hand muscles when assessed with TMS. One possible explanation can be attributed to the low capability of the BCI system to provide a timed association (cortical with the peripheral).

Therefore, there is still a gap to demonstrate the potential benefits of the combined use of neuromodulation BCI systems with assistive devices during the execution of functional tasks involving a multi-degree of freedom movement. In previous works, we presented a hybrid robotic system for rehabilitation of reaching movements in stroke patients [13]. This system is aimed to rehabilitate the arm motor impairment in stroke patients by executing unconstrained reaching movement in $3 \mathrm{D}$ space. In addition, an accurately and low latency EEG-based movement onset detection system was presented [14]. In this present study, the hybrid robotic system is combined with the low latency movement onset detection to verify whether the execution of functional reaching movement leads to a distributed plasticity (more than one muscle). Preliminary results of the combined system with healthy subjects are presented.

\section{METHODS}

\section{A. Subjects}

Five healthy subjects (three males and two females, age: 26.8 \pm 4.7 years) participated in this study. None of the subjects 
presented any history of sensory-motor disorder nor of physiological deficit.

\section{B. Hybrid robotic system}

The hybrid robotic system used in the experiments for this study was previously presented in [13], [15]. In brief, this system consists of three sub-blocks: the hybrid assistive block (a passive Exoskeleton and FES), the high-level control (HLC) block, and a visual feedback interface.

The hybrid assistance combines passive mechanical assistance on the upper limb through the ArmeoSpring ${ }^{\circledR}$ exoskeleton (Hocoma, Switzerland) and functional electrostimulation provided by the IntFES stimulator (Technalia, Spain). The exoskeleton supports the arm weight against gravity and allows carrying out $3 \mathrm{D}$ reaching movements in an unconstrained space. The electrical stimuli are delivered to the anterior deltoid (AD) and triceps (TR) muscles using biphasic electrical pulses.

The HLC block generates subject- and joint-specific reference trajectories and, by modulating the FES intensity, it provides the required support to perform the movements. This block was implemented in a PC104 architecture running the xPC Target operation system (Mathwork Inc) for real-time operation. The arm's reference trajectory was generated using a mathematical expression called minimum jerk [16], which assumes that the arm is moved smoothly from one point to another using a bell-shaped velocity profile. The feedback error learning (FEL) control algorithm was implemented to modulate the pulse width of the electrical signal applied over the arm's muscles (see [13], [15] for further details).

A cue-based visual feedback interface was developed to guide the users during the intervention (see Fig. 1). In addition, this interface provides information regarding the arm position (shoulder and elbow) and the task's performance. A state machine, depicted in Fig. 2, was implemented to manage the intervention's events and present the appropriate messages and icons to the users. This state machine relies on five main states: 'Wait', 'Relax', 'CountDown', 'Movement' and 'EndTrial'. During the 'CountDown' state, three different rectangles are displayed in the interface (see Fig. 1). These rectangles are incrementally filled up using a traffic light paradigm (red, yellow and green colors), lasting 1.5 seconds each. Thus, a period of 4.5 seconds is used for movement preparation. When the last rectangle is entirely filled (green color), all rectangles are turned in green, indicating the movement state. At this moment, the reference trajectory (denoted by the blue cross) start moving toward the target position (the red squared). The arm position is represented by the green circle, where the $\mathrm{x}$ - and $y$-axis indicate the movements of the elbow and shoulder joints, respectively. The system also reported information regarding the quality of the movement by lighting four different face's color after the movement execution according to the measured performance ('EndTrial state'). The green face indicated a good performance $(\geq 90 \%)$, yellow a moderate performance $(\geq 75$ $\%$ ), orange a bad performance $(\geq 50 \%)$ and red a poor performance $(\leq 50 \%)$. The visual feedback interface was coded using Matlab.

\section{EEG-Based BCI}

In order to tightly couple the subjects' intentions to move, a low-latency EEG-based BCI was used to control the onset of the assistance in each task performed. The BCI was implemented using real-time Simulink (The Mathworks Inc) on a dedicated computer. EEG signals were recorded from 28 positions $(\mathrm{AFz}$, F3-F4, FC3-FC4, C5-C6, CP3-CP4, P3-P4 and Oz according to the international 10-20 system) using active $\mathrm{Ag} / \mathrm{AgCl}$ electrodes (Acticap, Brain Products GmbH, Germany). The reference was set to the voltage of the earlobe contralateral to the affected arm and $\mathrm{Oz}$ was used as ground. Additionally, electromyography (EMG) signals were recorded using two bipolar electrodes placed at $\mathrm{AD}$ and TR muscles. EEG and EMG signals were amplified using the gUSBamp (g.Tecgmbh, Austria) and were sampled at $256 \mathrm{~Hz}$.

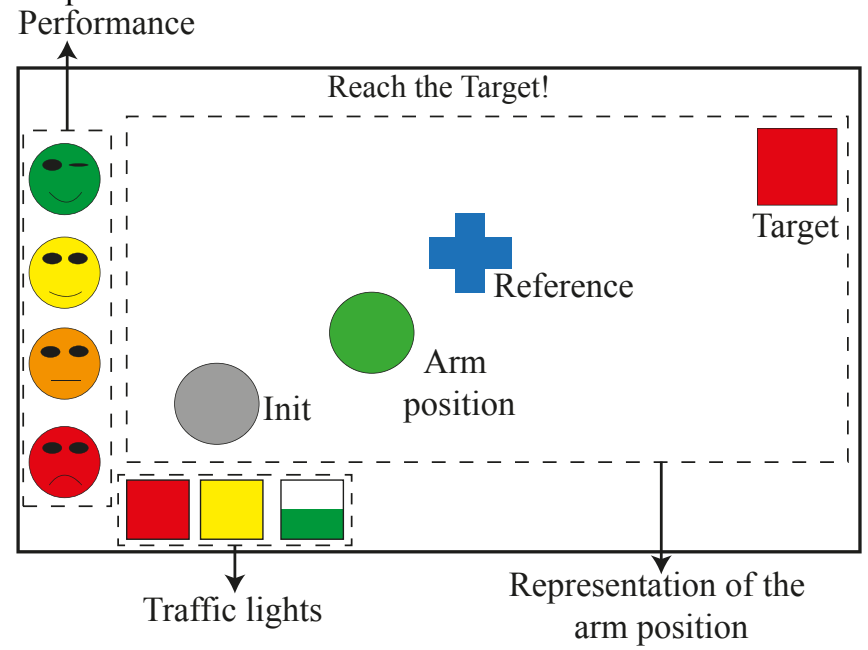

Fig. 1. Representation of the visual feedback interface.

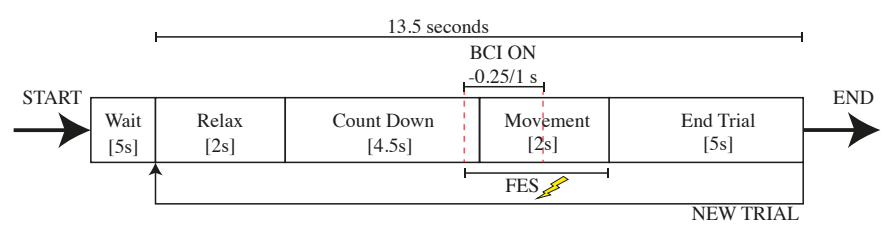

Fig. 2. Implemented state machine, representing the visual cue during the intervention.

The BCI system used to trigger the assistance based on the participant's movement intentions recorded from the EEG signals was the one presented in [14], [17]. The EMG signals from the $\mathrm{AD}$ and $\mathrm{TR}$ muscles were used to identify the movement onset and group the EEG signal into epochs (one for each movement). The acquired EMG signals were processed offline using a band-pass filter $(35 \geq \mathrm{f} \leq 120 \mathrm{~Hz})$ and a signal rectifier. A single threshold detector was implemented to detect onsets of muscle contractions using a threshold of $10 \%$ of the maximum EMG amplitude during the voluntary movements for each subject.

Two classifiers based on the event-related desynchronization (ERD) and the movement-related cortical potentials (MRCP) patterns were combined. A naïve Bayes classifier was used to detect the ERD patterns preceding the movements. Band-pass filtering (Butterworth, 2th order, $6 \leq \mathrm{f} \leq 30 \mathrm{~Hz}$ ) and a small Laplacian filter were applied. The power values were estimated using the Welch's method in segments of $1.5 \mathrm{~s}$ and for frequencies between 7-30 Hz (Hamming windows of $1 \mathrm{~s}, 50 \%$ overlapping). The values obtained in the calibration run (see 
section C.2) between $-3 \mathrm{~s}$ and $-0.5 \mathrm{~s}$ with respect to the movement onsets were labeled as resting state samples. Estimations generated at $\mathrm{t}=0 \mathrm{~s}$ were labeled as movement onset samples. The Bhattacharyya distance was used to select the 10 best features (channel/frequency pairs) to build the Bayesian classifier

The MRCP pattern was detected using a Butterworth bandpass filter (2th order, $0.05 \leq \mathrm{f} \leq 5 \mathrm{~Hz}$ ). A virtual channel was obtained by subtracting the average potential of channels F3, Fz, F4, C3, C4, P3, Pz and P4 to individually selected channels after a visual inspection. The average MRCP pattern from $-1.375 \mathrm{~s}$ to $0.125 \mathrm{~s}$ was obtained for the selected channels using the data obtained during the calibration phase. This pattern was then used to design a matched filter. During the online operation, the matched filter was applied to the virtual channel of the validation dataset.

Finally, the outputs from both detectors (ERD and MRCP) were combined using a logistic regression classifier. Finally, the outputs from both detectors (ERD and MRCP) were combined using a logistic regression classifier. To train this classifier samples of the resting and movement condition were taken from intervals between -3 and $-0.5 \mathrm{~s}$ (resting) and between -0.125 to $0.25 \mathrm{~s}$ (movement) with respect to the movement onsets. A threshold was applied to the estimations to decide every 19.53 ms (5 samples) whether a movement intention was detected. The threshold was optimally obtained using the calibration dataset and following the criterion of maximizing the true positive (TP) rate, i.e. the percentage of trials with a correct motor intention detection and with no incorrect detections.

\section{Experimental procedure}

The general protocol is depicted in Fig. 3. It consists of two main steps: assessment and intervention.

1. Pre-, post- and post30-assesment: The main outcome measure of the proposed intervention was the quantification of changes in the excitability of the motor cortex contralateral to the arm moved during the experiment. To this aim, the brain response to Transcranial Magnetic Stimulation (TMS) pulses delivered over the motor cortex at different intensities was measured from the AD and TR muscles before and after the intervention. For these assessments subjects were seated in a chair and they were instructed to keep their arms relaxed. After the skin preparation (rubbing with cotton and alcohol), EMG signals were recorded from the $\mathrm{AD}$ and $\mathrm{TR}$ muscles using surface electrodes (rectangular 22.225 x $34.925 \mathrm{~mm}$, NeuroPlus ${ }^{\mathrm{TM}}$ ) in a bipolar configuration. The ground electrode was placed over the wrist. In all subjects, the TMS stimuli were delivered on the right cerebral hemisphere in to induce Motor Evoked Potentials (MEPs) on the left arm. First, the hotspot of stimulation was determined. This site was identified as the area in which the most consistent MEPs on the AD and TR muscles were elicited simultaneously. This position was marked on the patient's head with a permanent marker to ensure that the stimuli were consistently delivered over the same area of the motor cortex before (pre-FES), after (post-FES) and 30 minutes after (post30-FES) the proposed intervention. Then, the subjectspecific resting motor threshold (rMT) was estimated using the reduced relative frequency method [18]. Next, MEPs were elicited in the resting arm at five different TMS intensities: $90 \%$, $100 \%, 110 \%, 120 \%$, and $130 \%$ of the rMT. Ten MEPs were recorded for each intensity and at each assessment stage. These ten MEPs were divided in two blocks of five stimuli each to avoid the possibility of biasing the measurements of a certain condition by misplacing the coil. The intensity of stimulation was randomized across subjects and delivered every 6.5 seconds.

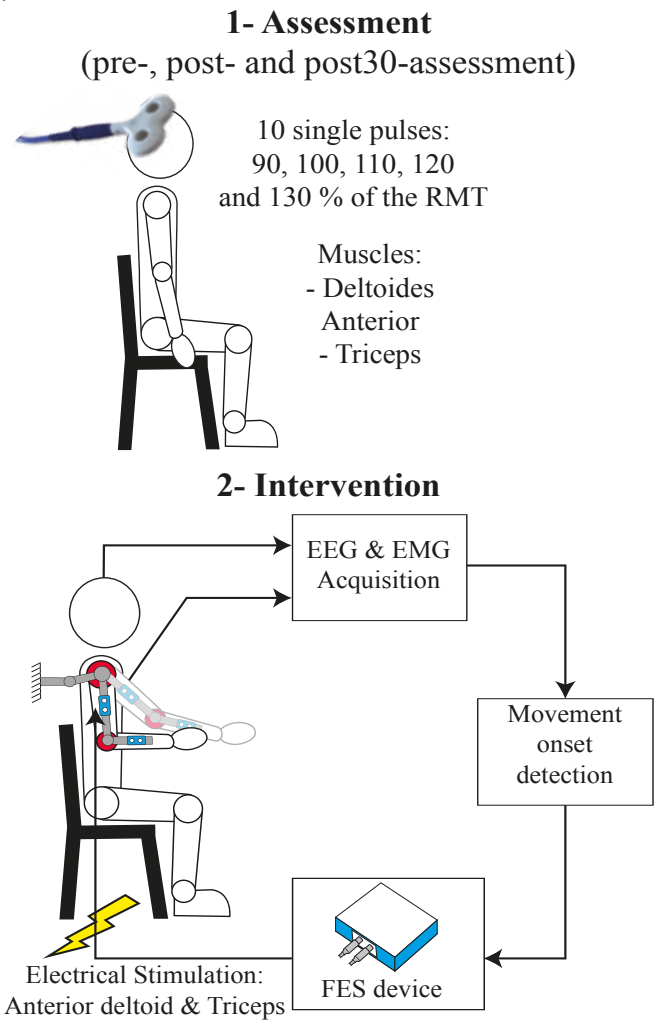

Fig. 3. Experimental protocol.

2. Intervention: the main part of the intervention consisted of a set of cue-guided reaching movements with the hybrid robotic assistance being triggered according to the subjects' motor intentions decoded from the EEG signals. Before starting the intervention, the subjects had to be equipped with assistive system. First, the ArmeoSpring was adjusted according to the subjects' arm lengths. The level of mechanical support was regulated such that the arm was kept about the subjects' thighs in the horizontal plane. Surface electrodes (Pals platinum rectangle $5 \times 5 \mathrm{~cm}$ ) were attached to the $\mathrm{AD}$ and TR muscles, and the maximum and minimum FES intensity was determined for each subject. Considering that the peripheral stimuli should be strong enough to recruit the afferent pathways and arrive at the motor cortex [8], [19], the minimum FES intensity was established as the amplitude in which the user perceived the stimulus and a visible muscle contraction was recognized (lightly below or equal to the motor threshold). The maximum FES intensity was defined as the maximum current that was considered comfortable by the subjects. The two thresholds were determined to assure that the FEL controller adjusted the current intensity within these range. After the calibration of the FES parameters, the subject-specific range of movement of the assisted limb was measured. This range of movement was used to define the maximum distance to be covered by the subject in each movement performed. 
Once the donning process was completed, the intervention proceeded with the calibration stage. At this stage, subjects were asked to follow the instructions represented in the visual feedback interface, which indicated them (using a countdown) when to perform reaching movements without FES. During the countdown, subjects were instructed to remain as relaxed and quite as possible. Each time the countdown was finished and the movement state was prompted, subjects were asked to perform the reaching movement following a reference trajectory displayed on the screen in front of them. A period of one second was allowed to reach the target from the initial position. All subjects carried out a total of 30 movements divided in two blocks of 15 movements each. The recorded data was used to train an EEG-based movement onset detector.

The last stage of the intervention consisted in the experimentation stage. At this stage, subjects repeated the execution of the same reaching movement carried out during the calibration, but electrical stimuli were delivered each time the EEG-based BCI detected the user own movement intention. To increase the accuracy of the BCI system and prevent subjects from receiving electrical stimuli wrongly, the output of the BCI system was enabled from $-250 \mathrm{~ms}$ to $+1 \mathrm{~s}$ with respect to the beginning of the 'Movement' state (see Fig. 2, vertical dotted lines in red). The subjects were advised that the electrical stimuli appeared whenever motor-related processes were observed within this window of time and they were instructed to report trials (movement executions) in which the FES was received too soon or too late with respect to their inner perception of their movement intentions and execution. All subjects carried out blocks of 20 movements until at least 50 good pairings (electrical stimuli arriving on time with the user's own intention to move) were achieved.

\section{E. Outcome measures and statistical analysis}

The main outcome measure was the change in MEP peak-topeak amplitude as a result of the intervention. A three-way repeated-measures ANOVA with factors 'Time' (1: pre; 2: post; and 3: post30) and 'Muscle' (1: AD; 2: TR) and 'Intensity' (1: $90 \% ; 2: 100 \% ; 3: 110 \% ; 4: 120 \%$; and 5: $130 \%)$ was used to investigate the effect of the intervention (BCI + hybrid robotic system) on the change in excitability in the descending corticomuscular pathway. Statistical significance was assumed if $\mathrm{P} \leq$ 0.05. In addition, the performance of the BCI system was assessed by estimating the TP rate according to the subjects' feedback. The TP rate was estimated from the experimentation phase of the intervention by computing the percentage of movements in which the subjects perceived the stimulus with their movement intentions.

\section{RESULtS}

The average rMT across all participants was $60.6 \pm 25.7 \%$ of the maximum stimulator output. All participants performed on average a total of $92 \pm 16.4$ intervention movements. The TP results of the BCI system was $77.3 \pm 12.6 \%$ across all subjects. The individual and general results are shown in table I.

Fig. 4 shows a representative example of the average MEPs amplitude for the AD and TR muscles of participant (P4) elicited at $120 \%$ of the rMT during the pre-, post- and post30assessment. It can be observed that the average peak-to-peak value of the MEP in the AD muscle increased $201 \%$ and $279 \%$ at the post- and post30-assessments with respect to the value of the pre-assessment condition. Similarly, the response measured in the TR muscle showed an increase of $243 \%$ and $148 \%$ at the post- and post30-assessment with respect to the pre-assessment. Similarly, the same pattern, an increased response after the intervention, was observed in the others TMS intensities.

TABLE I. PERFORMANCE OF THE CUE-BASED BCI SYSTEM

\begin{tabular}{|c|c|c|c|}
\hline Participants & $\begin{array}{c}\text { Total number of } \\
\text { movements }\end{array}$ & $\begin{array}{c}\text { Number of } \\
\text { Associations }\end{array}$ & $\begin{array}{c}\text { BCI accuracy } \\
\text { (TP) }\end{array}$ \\
\hline P1 & 110 & 52 & $62.72 \%$ \\
\hline P2 & 100 & 41 & $81 \%$ \\
\hline P3 & 70 & 51 & $80 \%$ \\
\hline P4 & 80 & 51 & $95 \%$ \\
\hline P5 & 100 & 48 & $68 \%$ \\
\hline Mean & $92 \pm 16.4$ & $48.6 \pm 4.5$ & $77.3 \pm 12.6 \%$ \\
\hline
\end{tabular}

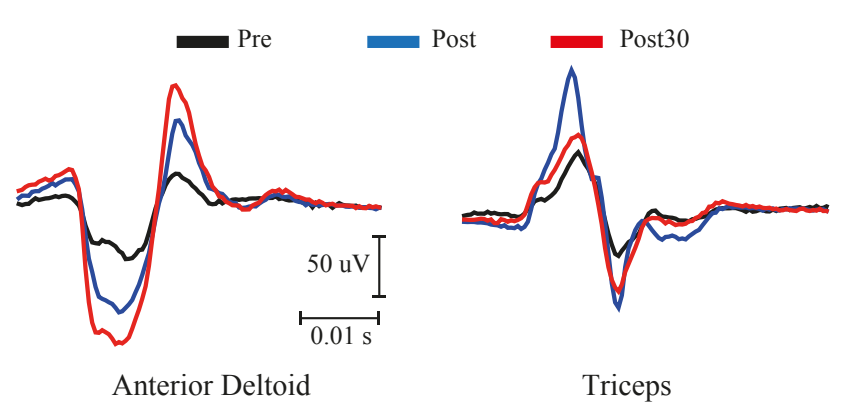

Fig. 4. A representative example of the elicited MEP prior and after the intervention.

Fig. 5 shows the averaged (across subjects) peak-to-peak values of the evoked MEPs prior to and following the interventions. In order to combined the results obtained with all subjects, these are expressed relative to the maximum peak-topeak value prior to the intervention. The statistical test did not find significant differences either when considering the threeway interaction ('Time' $\mathrm{x}$ 'Muscle' $\mathrm{x}$ 'Intensity', $\mathrm{P}=0.98$ ) or the two-way interaction ('Time' $\mathrm{x}$ 'Intensity', $\mathrm{P}=0.81$; 'Time' $\mathrm{x}$ 'Muscle', $\mathrm{P}=0.24)$, nor between the time intervals $(\mathrm{P}=0.19)$. Although no statistically significant differences were found, the trend of the responses was towards the increase of the corticospinal excitability as consequence of the intervention. In average, the AD peak-to-peak MEP at $130 \%$ of the rMT across subjects resulted in an increased of $6.9 \%$ and $13.1 \%$ in the postand post30-assessment with respect to the pre-assessment measurement. Likewise, the TR muscle reported an increase of $22.4 \%$ and $7.9 \%$ for the post- and post30-assessment respectively with respect to the pre-assessment.

\section{Discussion AND CONCLUSIONS}

To the authors' knowledge, this is the first study describing the modulation of cortical excitability by coupling voluntary motor-related cortical pattern with a hybrid robotic system during the execution of a functional task, involving a multidegree of freedom movement of the upper limb. Results showed an overall and distributed increase in the cortical excitability as a result of the proposed intervention. Although no significant differences were observed in the excitability of the cortical projection to the target muscles (AD and TR), it is expected that 
the changes observed here in five subjects become significant with a larger sample of subjects.

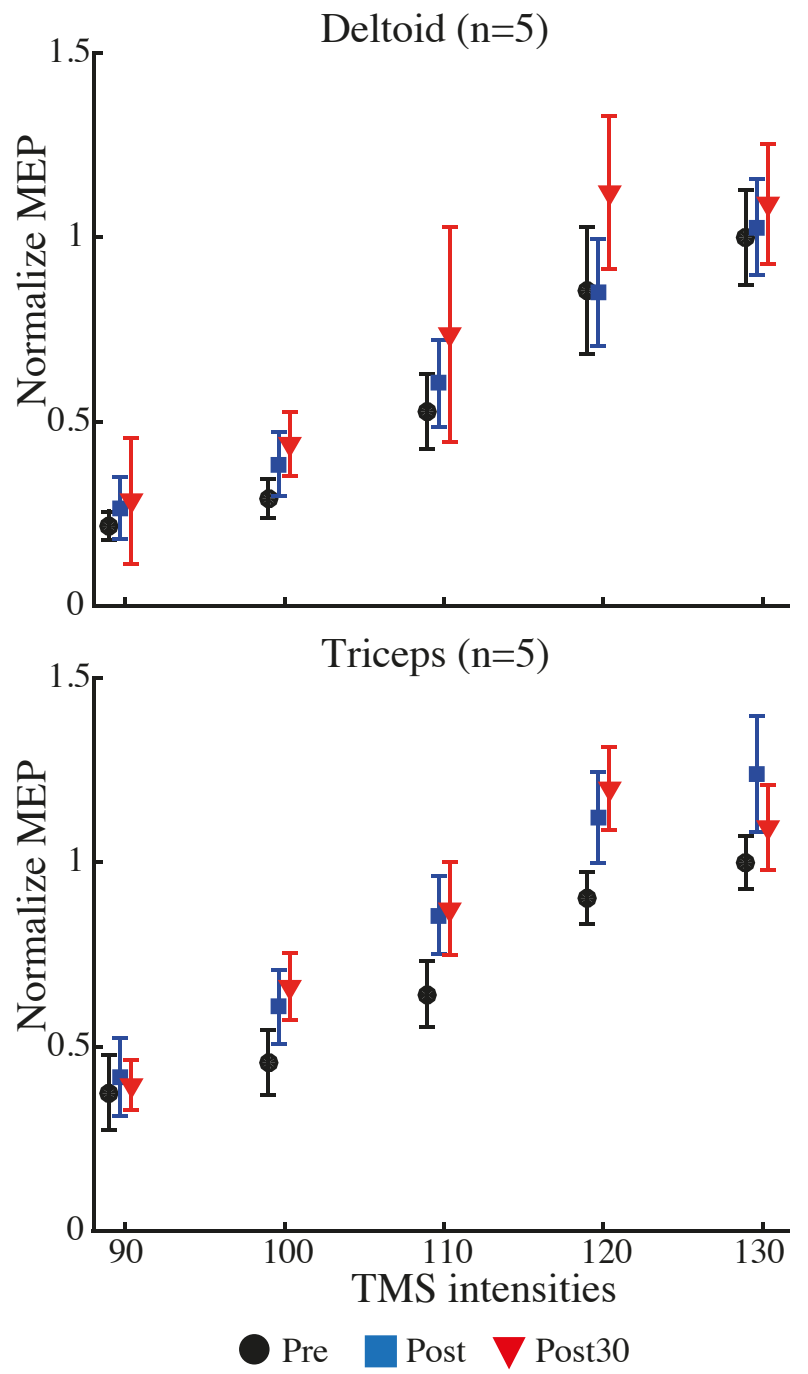

Fig. 5. Changes in descending corticospinal motor pathway excitability after the BCI intervention. Values represent the peak-to-peak value of the MEP before (pre), immediately after (post) and 30 minutes after the cessation of the intervention (post30) across all subjects. Error bars represent the $95 \%$ of confident intervals.

Robotic therapies and functional electrical stimulation have proven to be potentially beneficial to recover motor function after a brain damage. However, it is not clear under which circumstances robotic/FES therapies can maximize the functional benefit to the patients. Assuming that the recovery in patients with a stroke has to be achieved by reinforcing the cortical control of the paretic limb, it is expected that rehabilitation outcomes of robotic devices can be maximized when inducing plastic changes.

\section{REFERENCE}

[1] P. Maciejasz, J. Eschweiler, K. Gerlach-Hahn, A. Jansen-Troy, and S. Leonhardt, "A survey on robotic devices for upper limb rehabilitation.," $J$.
Neuroeng. Rehabil., vol. 11, p. 3, 2014.

[2] C. L. Lynch and M. R. Popovic, "Functional Electrical Stimulation," IEEE Control Syst. Mag., vol. 28, no. 2, pp. 40-50, Apr. 2008.

[3] F. Resquín, A. Cuesta Gómez, J. Gonzalez-Vargas, F. Brunetti, D. Torricelli, F. Molina Rueda, R. Cano de la Cuerda, J. C. Miangolarra, and J. L. Pons, "Hybrid robotic systems for upper limb rehabilitation after stroke: A review," Med. Eng. Phys., vol. 38, no. 11, pp. 1279-1288, Nov. 2016.

[4] J. W. Krakauer, "Motor learning: its relevance to stroke recovery and neurorehabilitation," Curr. Opin. Neurol., vol. 19, no. 1, pp. 84-90, 2006.

[5] P. Langhorne, J. Bernhardt, and G. Kwakkel, "Stroke rehabilitation," Lancet, vol. 377, no. 9778, pp. 1693-1702, 2011.

[6] J. J. Daly and J. R. Wolpaw, "Brain-computer interfaces in neurological rehabilitation," Lancet Neurol., vol. 7, no. 11, pp. 1032-1043, Nov. 2008.

[7] C. Ethier, J. Gallego, and L. Miller, "Brain-controlled neuromuscular stimulation to drive neural plasticity and functional recovery," Curr. Opin. Neurobiol., vol. 33, pp. 95-102, 2015.

[8] N. Mrachacz-Kersting, S. R. Kristensen, I. K. Niazi, and D. Farina, "Precise temporal association between cortical potentials evoked by motor imagination and afference induces cortical plasticity.," J. Physiol., vol. 590, no. Pt 7, pp. 1669-82, 2012.

[9] N. Mrachacz-Kersting, N. Jiang, A. J. T. Stevenson, I. K. Niazi, V. Kostic, A. Pavlovic, S. Radovanovic, M. Djuric-Jovicic, F. Agosta, K. Dremstrup, and D. Farina, "Efficient neuroplasticity induction in chronic stroke patients by an associative brain-computer interface.," J. Neurophysiol., 2015.

[10] R. Xu, N. Jiang, N. Mrachacz-Kersting, C. Lin, G. Asin Prieto, J. C. Moreno, J. L. Pons, K. Dremstrup, and D. Farina, "A closed-loop braincomputer interface triggering an active ankle-foot orthosis for inducing cortical neural plasticity," IEEE Trans. Biomed. Eng., vol. 61, no. 7, pp. 2092-2101, 2014.

[11] S. C. McGie, J. Zariffa, M. R. Popovic, and M. K. Nagai, "Short-Term Neuroplastic Effects of Brain-Controlled and Muscle-Controlled Electrical Stimulation," Neuromodulation Technol. Neural Interface, vol. 18, no. 3, pp. 233-240, 2015.

[12] D. Kraus, G. Naros, R. Bauer, M. T. Leão, U. Ziemann, and A. Gharabaghi, "Brain--robot interface driven plasticity: distributed modulation of corticospinal excitability," Neuroimage, vol. 125, pp. 522-532, 2016.

[13] F. Resquín, J. Gonzalez-Vargas, J. Ibáñez, F. Brunetti, and J. L. Pons, "Feedback error learning controller for functional electrical stimulation assistance in a hybrid robotic system for reaching rehabilitation.," Eur. $J$. Transl. Myol., vol. 26, no. 3, 2016.

[14] J. Ibáñez, J. I. Serrano, M. D. Del Castillo, E. Monge-Pereira, F. MolinaRueda, I. Alguacil-Diego, and J. L. Pons, "Detection of the onset of upper-limb movements based on the combined analysis of changes in the sensorimotor rhythms and slow cortical potentials," J. Neural Eng., vol. 11, no. 5, p. 56009, 2014.

[15] F. Resquín, J. Ibáñez, J. Gonzalez-Vargas, F. Brunetti, I. Dimbwadyo, S. Alves, L. Carrasco, L. Torres, and J. L. Pons, "Combining a hybrid robotic system with a bain-machine interface for the rehabilitation of reaching movements: A case study with a stroke patient," in 2016 38th Annual International Conference of the IEEE Engineering in Medicine and Biology Society (EMBC), 2016, pp. 6381-6384.

[16] T. Flash and N. Hogan, "The coordination of arm movements: an experimentally confirmed mathematical model.," J. Neurosci., vol. 5, no. 7, pp. $1688-1703,1985$.

[17] J. Ibáñez, E. Monge-Pereira, F. Molina-Rueda, J. I. Serrano, M. D. del Castillo, A. Cuesta-G?mez, M. Carratal?-Tejada, R. Cano-de-la-Cuerda, I. M. Alguacil-Diego, J. C. Miangolarra-Page, and J. L. Pons, "Low Latency Estimation of Motor Intentions to Assist Reaching Movements along Multiple Sessions in Chronic Stroke Patients: A Feasibility Study," Front. Neurosci., vol. 11, Mar. 2017.

[18] S. Groppa, A. Oliviero, A. Eisen, A. Quartarone, L. G. Cohen, V. Mall, A. Kaelin-Lang, T. Mima, S. Rossi, G. W. Thickbroom, P. M. Rossini, U. Ziemann, J. Valls-Solé, and H. R. Siebner, "A practical guide to diagnostic transcranial magnetic stimulation: Report of an IFCN committee," Clin. Neurophysiol., vol. 123, no. 5, pp. 858-882, May 2012.

[19] K. Stefan, E. Kunesch, L. G. Cohen, R. Benecke, and J. Classen, "Induction of plasticity in the human motor cortex by paired associative stimulation," Brain, vol. 123, pp. 572-584, 2000. 